\title{
Sistema OFDM com estimadores de frequência e de fase para sincronização de portadora
}

\section{OFDM system with frequency and phase estimators for carrier synchronization}

\section{Sistema OFDM con estimadores de frecuencia y de fase para sincronización de portadora}

\section{Système OFDM avec des estimateurs de fréquence et de phase pour la synchronisation de la porteuse}

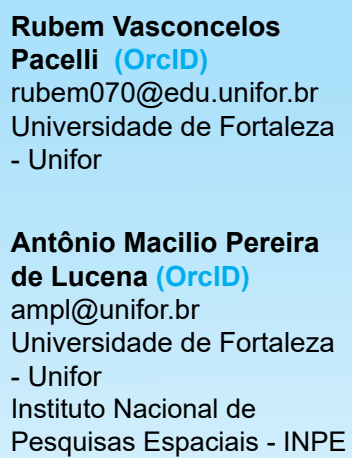

Antônio Macilio Pereira de Lucena (OrcID) ampl@unifor.br Universidade de Fortaleza - Unifor Instituto Nacional de Pesquisas Espaciais - INPE

\begin{abstract}
Resumo
Orthogonal frequency-division multiplexing, ou OFDM, é uma técnica de modulação para comunicações sem fio em que se utilizam múltiplas portadoras ortogonais com alguma superposição espectral. Apesar de sua grande robustez contra multipercuso em canais móveis, o sistema OFDM apresenta alta susceptibilidade a erros de sincronismos de frequência e de fase. O presente artigo apresenta uma arquitetura de comunicação em que o receptor é dotado de estimadores de fase e frequência que, em conjunto, mitigam os efeitos dos erros de frequência e de fase. O desempenho do sistema é analisado em termos da taxa de erro de bits. Adicionalmente, é investigada a performance do sistema quando cada um dos estimadores opera isoladamente. Também é avaliado o desempenho dos estimadores em termos da variância do erro dos parâmetros estimados e comparado com o limite de Cramer-Rao. Como principal resultado, verificou-se que o sistema, quando funcionando com os dois estimadores simultaneamente, cancela os erros de sincronismo de frequência e de fase e atinge uma taxa de erro de bits próximo do limite teórico.
\end{abstract}

Palavras-chave: OFDM; Sincronismo de portadora. CFO. ADPLL. Malha de Costa.

\begin{abstract}
Orthogonal frequency-division multiplexing, or OFDM, is a modulation technique used in wireless communications in which multiple orthogonal carriers with some spectral superposition are used. Despite its great robustness against multipath in mobile channels, the OFDM system presents high susceptibility to frequency and phase synchronization errors. The present paper presents a OFDM communication system in which the receiver is equipped with phase and frequency estimators that, together, mitigate the effects of frequency and phase errors. The performance of the system is analyzed in terms of the bit error rate. In addition, the performance of the system is investigated when each of the estimators operates separately. We also evaluate the performance of the estimators in terms of the variance of the error of the estimated parameters and compared with the Cramer-Rao limit. As a main result, it was found that, when operating with both estimators simultaneously, the system cancels the frequency and phase sync errors and reaches a bit error rate close to the theoretical limit.
\end{abstract}

Keywords: OFDM. Carrier sinchronization. CFO. ADPLL. Costa loop. 


\title{
Resumen
}

Orthogonal frequency-division multiplexing, o OFDM, es una técnica de modulación utilizada en comunicaciones inalámbricas en la cual se utilizan múltiples portadoras ortogonales con alguna superposición espectral. Aunque tenga gran robustez contra multipercurso en canales móviles, el sistema OFDM presenta alta susceptibilidad a errores de sincronismos de frecuencia y de fase. Este artículo presenta una arquitectura de comunicación en que el receptor es dotado de estimadores de fase y frecuencia que, en conjunto, mitigan los efectos de los errores de frecuencia y de fase. El rendimiento del sistema es analizado en términos de la tasa de error de bits. Adicionalmente, se investiga el desempeño del sistema cuando cada uno de los estimadores opera aisladamente. También se evalúa el rendimiento de los estimadores en términos de la variación del error de los parámetros estimados y comparado con el límite de Cramer-Rao. Como principal resultado, se verificó que el sistema, cuando está funcionando con los dos estimadores a la vez, se cancelan los errores de sincronismo de frecuencia y de fase y se alcanza una tasa de error de bits próximo del límite teórico. Como principal resultado, verificou-se que o sistema, quando funcionando com os dois estimadores simultaneamente, cancela os erros de sincronismo de frequência e de fase e atinge uma taxa de erro de bits próximo do limite teórico.

Palabras-clave: OFDM. Sincronismo de portadora. CFO. ADPLL. Malla de Costa.

\begin{abstract}
Résumé
Orthogonal frequency-division multiplexing, ou OFDM, est une technique de modulation utilisée dans des communications sans fil où s'utilisent de multiples sous-porteuses orthogonales avec quelque superposition spectrale. Malgré sa grandeur contre multitrajet dans des canaux mobiles, le système OFDM a de la susceptibilité à des erreurs de synchronismes de fréquence et de phase. Cet article présente une architecture de communication où le récepteur est doté d'estimateurs de phase et de fréquence qui atténuent ensemble les effets des erreurs de fréquence et de phase. De la Performance du système est analysée en termes de taux d'erreur sur les bits. En outre, de la performance du système est analysée quand chacun des estimateurs fonctionne tous seuls. De la performance des indicateurs est aussi évaluée en fonction de la variation de l'erreur des paramètres estimés et comparés à la borne Cramer-Rao. Comme principal résultat, il s'est vérifié que le système annule les erreurs de synchronisme de fréquence et de phase et atteint un taux d'erreur sur les bits proche du limite théorique, quand il fonctionne avec les deux estimateurs simultanément
\end{abstract}

Mots-clés: OFDM. Synchronisme de la porteuse. CFO. ADPLL. Boucle de Costas.

\section{Introdução}

Orthogonal frequency division multiplexing, ou OFDM, é uma técnica de transmissão que surgiu em 1968, desenvolvida por Chang e Gibby, e foi patenteada, posteriormente, no Estados Unidos (WEINSTEIN, Stephen B. 2009). O sistema OFDM emprega multiportadoras, as quais se sobrepõem no domínio da frequência, possibilitando assim uma alta eficiência espectral. Nessa técnica de transmissão, um canal seletivo na frequência é dividido em subcanais não seletivos na frequência, em que se alocam cada subportafora que transmite com uma taxa menor.

O sincronismo de portadoras em OFDM é uma etapa crucial para que o receptor funcione de modo satisfatório e a sua falta degrada fortemente o desempenho do sistema (MA, Xiaoqiang; KOBAYASHI, Hisashi, 2003). Devido à própria natureza física inerente de cada oscilador é praticamente impossível que transmissor e o receptor estejam em perfeita sincronia, havendo sempre um desvio de frequência. Definindo o desvio total de frequência experimentado pelo sistema como $f_{\text {offset }}=f_{t}-f_{r}$, em que $f_{t} e f_{r}$ são a frequência do sinal transmitido e recebido, respectivamente, e o desvio de frequência normalizada $\varepsilon$ para um sistema OFDM é dado por (PRIYA, C. G, 2013): 
sendo $\Delta f$ o espaçamento de frequência entre as subportadoras.

$$
\varepsilon=\frac{f_{\text {offset }}}{\Delta f}
$$

Levando em conta o desvio em frequência, o sinal no receptor com $N$ subportadoras é (CHO, 2010):

$$
y[n]=\frac{1}{N} \sum_{m=0}^{N-1} H[m] X[m] e^{j \frac{2 \pi}{N} n(m+\varepsilon)},
$$

em que $H[m]$ e $X[m]$ representam a resposta em frequência do canal e o sinal discreto transmitido, respectivamente. Sendo $Y[k]$ o sinal discreto recebido no domínio da frequência, temos:

$$
Y[k]=\sum_{n=0}^{N-1} y[n] \cdot e^{-j \frac{2 \pi}{N} n k} .
$$

Substituindo a Eq. (2) na Eq. (3) e fazendo-se alguns cálculos algébricos, obtém-se:

$$
\begin{aligned}
& Y[k]=\frac{1}{N} \sum_{n=0}^{N-1} \sum_{m=0}^{N-1} H[m] \cdot X[m] \cdot e^{j \frac{2 \pi}{N} n(m+\varepsilon)} \cdot e^{-j \frac{2 \pi}{N} n k}, \\
& Y[k]=\frac{1}{N} H[k] \cdot X[k] \sum_{n=0}^{N-1} e^{j \frac{2 \pi}{N} n \varepsilon}+\frac{1}{N} \sum_{m=0, m \neq k}^{N-1} H[m] . X[m] \sum_{n=0}^{N-1} \cdot e^{j \frac{2 \pi}{N} n(m+\varepsilon-k)},
\end{aligned}
$$

ou ainda:

$$
Y[k]=\frac{\sin \pi \varepsilon}{N \cdot \sin \frac{\pi \varepsilon}{N}} . H[k] X[k] e^{j \frac{\pi \varepsilon(N-1)}{N}}+e^{j \frac{\pi \varepsilon(N-1)}{N}} \sum_{m=0, m \neq k}^{N-1} \frac{\sin (\pi(m-k-\varepsilon))}{N \sin (\pi / N(m-k-\varepsilon))} H[m] X[m] e^{j \frac{\pi \varepsilon(m-k)(N-1)}{N}} .
$$

Na equação (6), o primeiro termo (fazendo $m=k$ ) é o sinal de interesse, enquanto que o segundo termo se refere à interferência das demais portadoras. Observe que o desvio de $\varepsilon$ faz com que se perca a ortogonalidade entre as mesmas. Da equação (6), podemos concluir que, devido ao efeito de desvio em frequência, o sinal recebido é rotacionado e amplificado, além do resíduo do segundo termo da equação, derivado da quebra da ortogonalidade das portadoras (LI, 2001). Para exemplificar o efeito do CFO (do inglês, Carrier Frequency Offset), são mostradas nas fig. 1 e fig. 2 as constelações dos sinais recebidos com relação sinal-ruído de $10 \mathrm{~dB}$, para um sistema sem desvio em frequência e para um sistema com desvio em frequência $(\varepsilon=0,1)$, respectivamente. 
Figura 1 - Constelação dos sinais recebidos para um sistema sem o efeito do desvio de frequência para

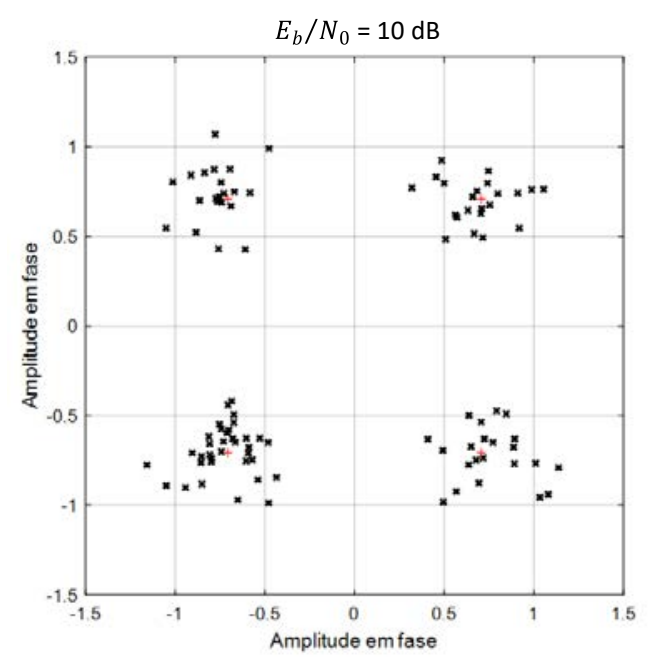

Fonte: Elaborado pelos próprios autores (2018).

Figura 2 - Constelação dos sinais recebidos para um sistema com o efeito do desvio de frequência para

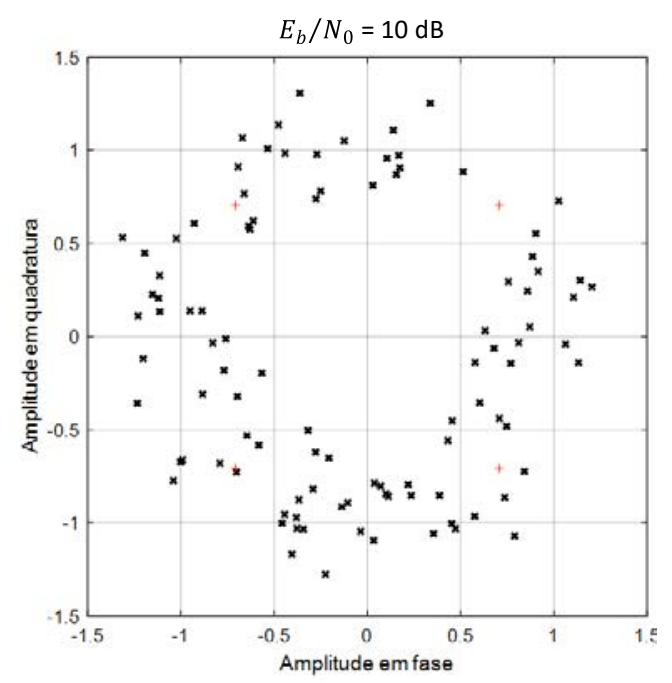

Fonte: Elaborado pelos próprios autores (2018).

É possível notar que, com o desvio em frequência, a constelação dos sinais recebidos fica bastante distorcida, enquanto o sistema sem o desvio em frequência, sob as mesmas condições, permanece com sua constelação sem grandes alterações. Além disso, é possível notar que houve uma rotação dos sinais transmitidos, validando o foi previsto na eq. (6) (MA, 2003).

Este artigo propõe uma arquitetura de sincronização para sistema OFDM que corrige o efeito de desvio de frequência através de duas operações independentes entre si. Um estimador de offset de frequência, operando em malha aberta, que faz a correção grosseira do desvio de frequência, enquanto um estimador de fase, operando em malha fechada, corrige o desvio de fase e consequentemente anula algum offset de frequência residual. $O$ desempenho do sistema em termos de taxa de erro de bits para diversas condições de desvio de frequência é avaliado através de simulação computacional e os resultados são apresentados e 
discutidos. Apresentam-se também gráficos da variância dos estimadores de fase e frequência comparados com os limites teóricos.

O trabalho está organizado da seguinte forma: Na secção 2 se apresentam a descrição do sistema e o detalhamento do projeto dos sincronizadores. Por meio de diagrama de bloco e equações, detalha-se o funcionamento do sistema como um todo e de seus módulos funcionais. Os resultados das simulações computacionais para verificação da performance do sistema, com algumas discussões, são apresentados na secção 3. Na secção 4, conclui-se o artigo.

\section{Descrição do Sistema}

\subsection{Visão Geral}

Neste trabalho, adota-se o modelo discreto equivalente do sistema OFDM nas descrições e nas análises. A figura 3 mostra o diagrama de blocos do sistema visto como uma associação de transmissor, canal e receptor. Os dados binários $b[i]$ e $\left\{\begin{array}{ll}0 & 1\end{array}\right\}$ são entregues ao transmissor OFDM, sendo gerado o sinal OFDM a ser transmitido, representado por $q[n]$.

Figura 3 - Modelo discreto equivalente do sistema de comunicação.

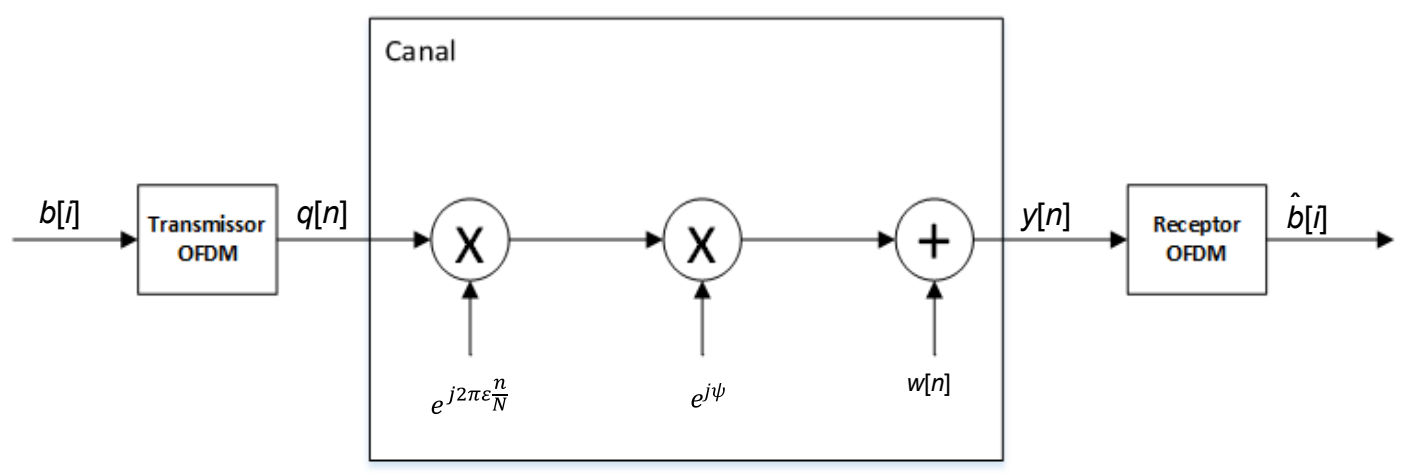

Fonte: Elaborado pelos próprios autores (2018).

Ao passar pelo canal, o sinal transmitido sofre desvio de frequência e de fase, representado por $e^{j 2 \pi \varepsilon \frac{n}{N}}$ e $e^{j \psi}$, respectivamente, em que indica uma fase desconhecida no intervalo de 0 a 2 . Além disso, o sinal é distorcido pelo ruído AWGN (do inglês, Additive White Gaussian Noise), representado por w[n], que é um processo gaussiano discreto, branco, complexo, com média nula e densidade espectral de potência $2 N_{0}$.

Após sofrer as distorções do canal, o sinal recibo $y[n]$ entra no receptor OFDM, que recupera as informações dos dados binários transmitidos, denotadas por . No sistema proposto, o receptor OFDM é dotado de estimadores de fase e frequência para a correção dos desvios causados pelo canal.

\subsection{Transmissor OFDM}

O diagrama de blocos do transmissor OFDM é mostrada na fig. 4.

Figura 4 - Transmissor OFDM

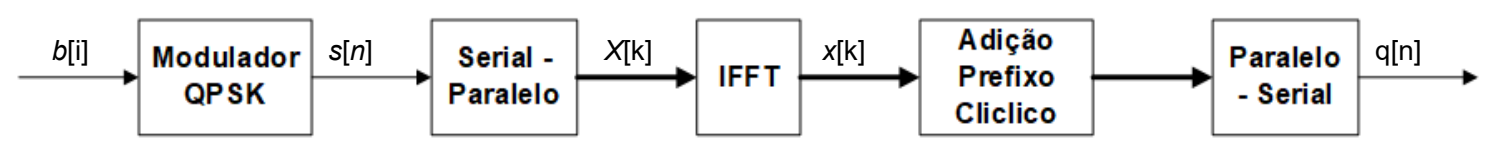

Fonte: Elaborado pelos próprios autores (2018). 
Os dados binários transmitidos $b[i]$ são modelados por uma variável aleatória, com valores lógicos 0 ou 1, com probabilidade de 1/2 e gerados a uma taxa 2/T. O modulador QPSK (do inglês, Quadrature phase shift keying) faz o mapeamento de cada par de bits, em um símbolo complexo $s[n]$ da constelação QPSK, resultando em uma sequência de símbolos na taxa $1 / T$.

Em seguida, o bloco "Serial-paralelo" converte os símbolos seriais $s[n]$ em um símbolo paralelo composto por $N$ subsímbolos, representado pelo vetor:

$$
\boldsymbol{X}[k]=\left[X_{0}[k], X_{1}[k] \ldots X_{N-1}[k]\right],
$$

em que $k$ representa o índice temporal do $k$-ésimo símbolo e a $q$-ésima componente do vetor é dada por:

$$
X_{q}[k]=s[N k+q] .
$$

Depois da conversão, o símbolo X[k] é transformado em pelo bloco IFFT (do inglês, Inverse Fast Fourier transform), conforme a seguinte operação matemática:

$$
x_{m}[k]=\frac{1}{N} \sum_{p=0}^{N-1} X_{p}[k] e^{j \frac{2 \pi}{N} p m}, \quad 0 \leq m \leq N-1 .
$$

O bloco "Adição de prefixo cíclico" modifica o símbolo $\boldsymbol{x}[k]$, para criar um novo símbolo denotado por e expresso $\widetilde{x}[k]$ pela equação:

$$
\widetilde{\boldsymbol{x}}[k]=\left[x_{N-1-N_{g}}[k], x_{N-1-N_{g}+1}[k] \ldots x_{N-1}[k], x_{0}[k], x_{1}[k] \ldots x_{N-1}[k]\right],
$$

em que $\mathrm{N}_{\mathrm{g}}$ indica o comprimento do prefixo cíclico. Note que $\widetilde{\boldsymbol{x}}[k]$ é um vetor de dimensão $\mathrm{N}+\mathrm{N}_{\mathrm{g}}$ que contém todas as componentes do vetor $x[k]$.

Por fim, o bloco "Paralelo-serial" realiza a operação inversa do bloco "Serial-paralelo", transformando o $\widetilde{x}[k]$ em um sinal serial, representado por $q[n]$. Portanto, na saída do transmissor tem-se uma sequência serial de amostras do sinal $q\lfloor n\rfloor$ a uma taxa $1 / T$.

\subsection{Receptor OFDM}

A figura 5 mostra o esquemático do receptor OFDM.

Figura 5 - Receptor OFDM

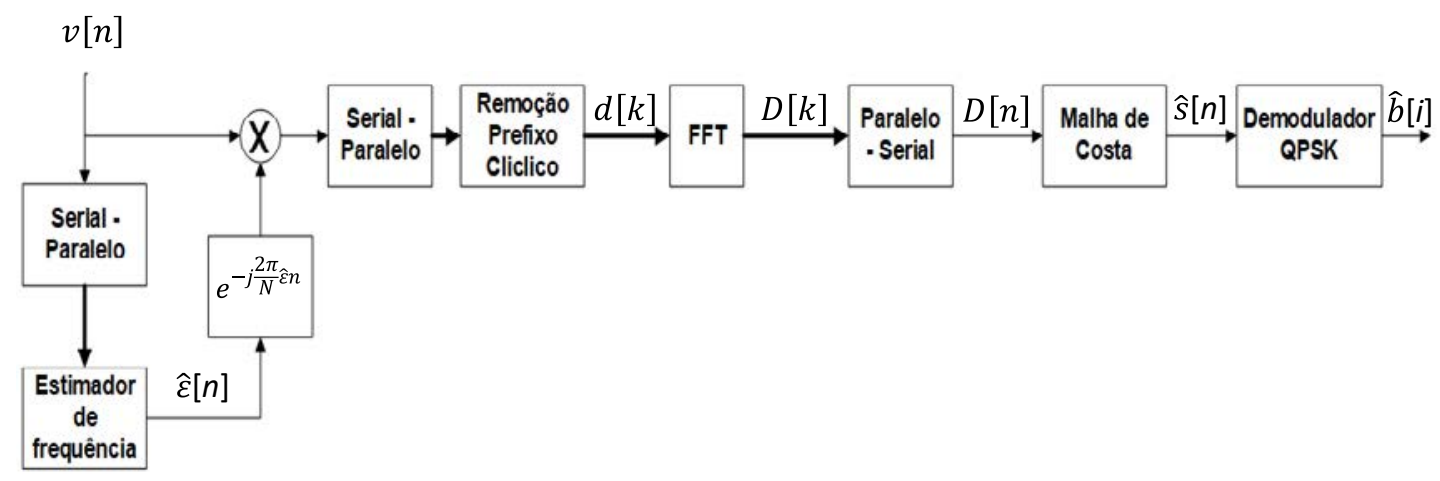

Fonte: Elaborado pelos próprios autores (2018).

Após o sinal OFDM transmitido $q[n]$ passar pelo canal, é obtido o sinal OFDM recebido, $y[n]$. A operação para obter o sinal $y[n]$ é mostrada na fig. 3. Com base nessa figura, pode-se concluir que:

$$
y[n]=q[n] e^{j\left(\frac{2 \pi}{N} n \varepsilon+\psi\right)}+w[n] .
$$


A arquitetura do receptor incorpora um estimador de frequência e um estimador de fase que, operando em conjunto, mitigam os efeitos de eventuais CFO presentes no sistema.

A partir do sinal recebido, como indicado na fig. 5 , o estimador de frequência determina o valor de $\hat{\varepsilon}$ que representa a estimativa do desvio de frequência $\varepsilon$. Um oscilador local gera o sinal $e^{-\frac{2 \pi n}{N} \hat{\varepsilon}}$ que, ao ser multiplicado pelo sinal de entrada $y[n]$, corrige parcialmente o desvio de frequência. A estimativa é feita pelo módulo estimador de frequência a partir da equação (WU, 2007; MORELLI, 2007):

$$
\widehat{\varepsilon}=<\frac{1}{2 \pi} \arg \left\{\sum_{n=-N_{g}}^{-1} \mathrm{y}^{*}[n] y[n+N]\right\}>_{K},
$$

em que o operador $\left\langle x>K\right.$ representa a média temporal móvel de $x$ utilizando $K$ termos, $c^{*}$ indica o conjugado de $c$ e $\mathrm{N}_{\mathrm{g}}$ é o comprimento do prefixo cíclico. Para cada símbolo OFDM recebido, determina-se um novo conjunto de valores para o argumento do somatório da eq. (12), e esse valor é utilizado para o cálculo da média que vai resultar em $\hat{\varepsilon}$. Devido à natureza ruidosa do sinal $y[n]$, a estimativa do CFO não é perfeita e, por consequência, $\varepsilon-\hat{\varepsilon} \neq 0$. Portanto um pequeno CFO residual, igual $\varepsilon-\hat{\varepsilon}$, é passado às etapas seguintes do receptor OFDM.

Com o sinal parcialmente corrigido, a arquitetura do receptor realiza as operações inversas àquelas feitas na transmissão. O bloco "Serial-paralelo" paraleliza o sinal recebido em $N+N_{g}$ subsímbolos, e o bloco "Remoção prefixo cíclico" descarta os primeiros $N_{g}$ subsímbolos, resultando no sinal paralelo $d[k]$ com $N$ subsímbolos expresso por:

$$
\boldsymbol{d}[k]=\left[d_{0}[k], d_{1}[k] \ldots d_{N-1}[k]\right] .
$$

O bloco "FFT" transforma os $N$ subsímbolos do sinal paralelo d[k] no sinal:

$$
\boldsymbol{D}[k]=\left[D_{0}[k], D_{1}[k] \ldots D_{N-1}[k]\right]
$$

através da transformada rápida de Fourier, ou FFT(do inglês, Fast Fourier transform), dada pela seguinte equação:

$$
D_{p}[k]=\sum_{m=0}^{N-1} d_{m}[k] e^{-j \frac{2 \pi}{N} m p}, \quad 0 \leq p \leq N-1,
$$

em que $D_{p}[k]$ indica o subsímbolo obtido após a FFT. No bloco "Paralelo-serial", os subsímbolos são serializados, gerando o sinal D[n], que pode ser expresso por (MEYR H. et al., 1998):

$$
D[n]=s[n] e^{j \theta[n]}+z[n],
$$

em que $s[n]$ é o símbolo QPSK transmitido, $z[n]$ é a parcela de ruído, que modelado como sendo , branco, complexo, com média nula e densidade espectral $2 N_{0}$, e $\theta[n]$ é o desvio fase decorrente do offset de frequência residual não corrigido e do erro de fase $\psi$ introduzido pelo canal. Essa fase é modelada pela seguinte expressão:

$$
\theta[n]=\frac{2 \pi(\varepsilon-\hat{\varepsilon})}{N} n+\psi
$$

O bloco denominado de "Malha de costa" é o estimador de fase do receptor, que tem como objetivo corrigir as distorções de fase do sinal $s[n]$ transmitido. O diagrama de blocos da Malha de Costa discreta implementada é mostrado na fig. 6 (LUCENA, 2017). 
Figura 6 - Diagrama de blocos da Malha de Costa.

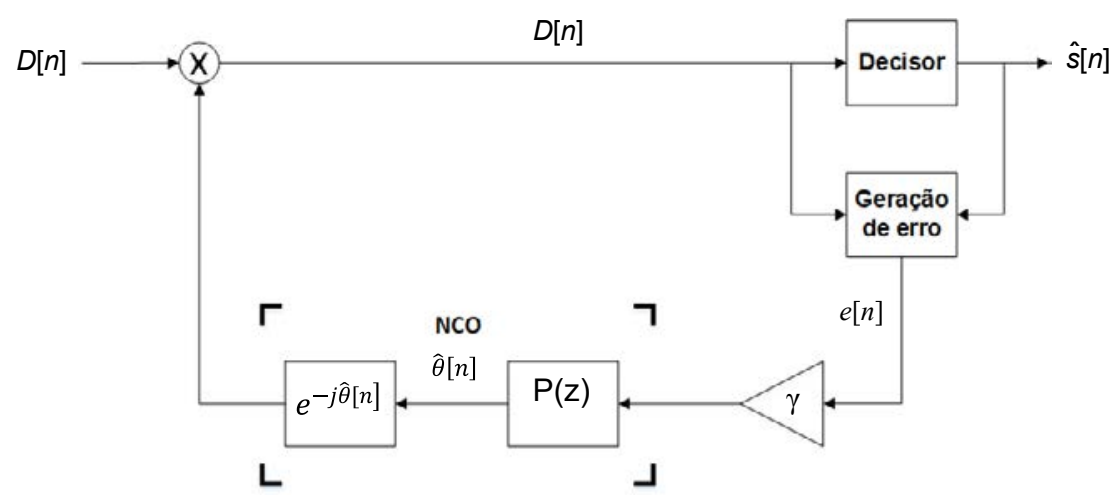

Fonte: Elaborado pelos próprios autores (2018).

A malha faz uma estimativa da fase $\theta[n]$, indicada no diagrama por $\hat{\theta}[n]$, e efetua a correção necessária no sinal $D[n]$ através da multiplicação por $e^{-j \hat{\theta}[n]}$, gerando o sinal corrigido $\widehat{D}[n]$. O bloco "Decisor" realiza a deteç̧ão do símbolo QPSK recebido, originando o sinal $\hat{s}[n]$, que representa uma estimativa do sinal $s[n]$ transmitido.

Desconsiderando o ruído gaussiano e supondo $\hat{s}[n]$ a decisão correta, o sinal de erro é dado por:

$$
e[n]=\frac{\operatorname{Im}\left\{\widehat{D}[n] \hat{s}^{*}[n]\right\}}{2}=\operatorname{sen}\{\theta[n]-\hat{\theta}[n]\},
$$

Para o caso em que $\theta[n]-\hat{\theta}[n]<<1$, a expressão para o erro se reduz a:

$$
e[n]=\theta[n]-\hat{\theta}[n] .
$$

O filtro de malha é constituído apenas pelo ganho (controlador proporcional), que representa o passo de adaptação do estimador. O NCO (do inglês, Numerically Controlled Oscillator), que tem como função gerar a exponencial $e^{-j \hat{\theta}[n]}$ a partir do sinal de erro e[n] (SHAYAN, Y. R., 1989), é implementado pelo integrador $P(z)$ e uma tabela de busca cuja saída é a exponencial complexa. Definindo a transformada $z$ bilateral de um dado sinal $a[n]$, como:

$$
A(z)=\sum_{n=-\infty}^{\infty} a[n] z^{-n},
$$

A função de sistema do integrador do NCO, indicada por $P(z)$ (LINDSEY, William C., 1981), é:

$$
P(z)=\frac{z^{-1}}{1-z^{-1}} \text {. }
$$

Portanto, a transformada $z$ da estimativa de fase $\hat{\theta}(z)$ é:

$$
\hat{\theta}(z)=\gamma E(z) P(z),
$$

em que o erro $E(z)$ é expresso por:

$E(z)=\theta(z)-\hat{\theta}(z)$

Substituindo a eq. (23) e eq. (21) na eq. (22), obtém-se a função de sistema da malha inteira, dada por:

$$
F(z)=\frac{\hat{\theta}(z)}{\theta(z)}=\frac{\gamma z^{-1}}{1+(\gamma-1) z^{-1}}
$$


Pode-se expressar a transformada $z$ do sinal de erro em termos da função de sistema a malha. Logo, da eq. (23), têm-se:

$$
E(z)=\theta(z)-F(z) \theta(z)=\theta(z)(1-F(z)) .
$$

Para analisar como a malha se comporta diante de um desvio de fase, determina-se a seguir a expressão do sinal de erro, $e[n]$ e $E(z)$, quando se aplica um degrau de fase de amplitude $\Delta \theta$ :

$$
\theta[n]=\Delta \theta u[n] .
$$

Realizando a transformada z na eq. (26) e isolando o desvio de fase, obtêm-se:

$$
\theta(z)=\Delta \theta \frac{1}{1-z^{-1}} \text {. }
$$

Substituindo a eq. (27) e (24) na eq. (25), têm-se que:

$$
E(z)=\Delta \theta \frac{1}{1-(1-\gamma) z^{-1}} \text {. }
$$

A transformada $z$ inversa da eq. (28) resulta em:

$e[n]=\Delta \theta(1-\gamma)^{n}$.

A figura 7 mostra a resposta transitória do sinal de erro para diferentes valores de normalizado por .

Figura 7 - Sinal de erro para diferentes valores de $y$ normalizado por $\Delta \theta$.

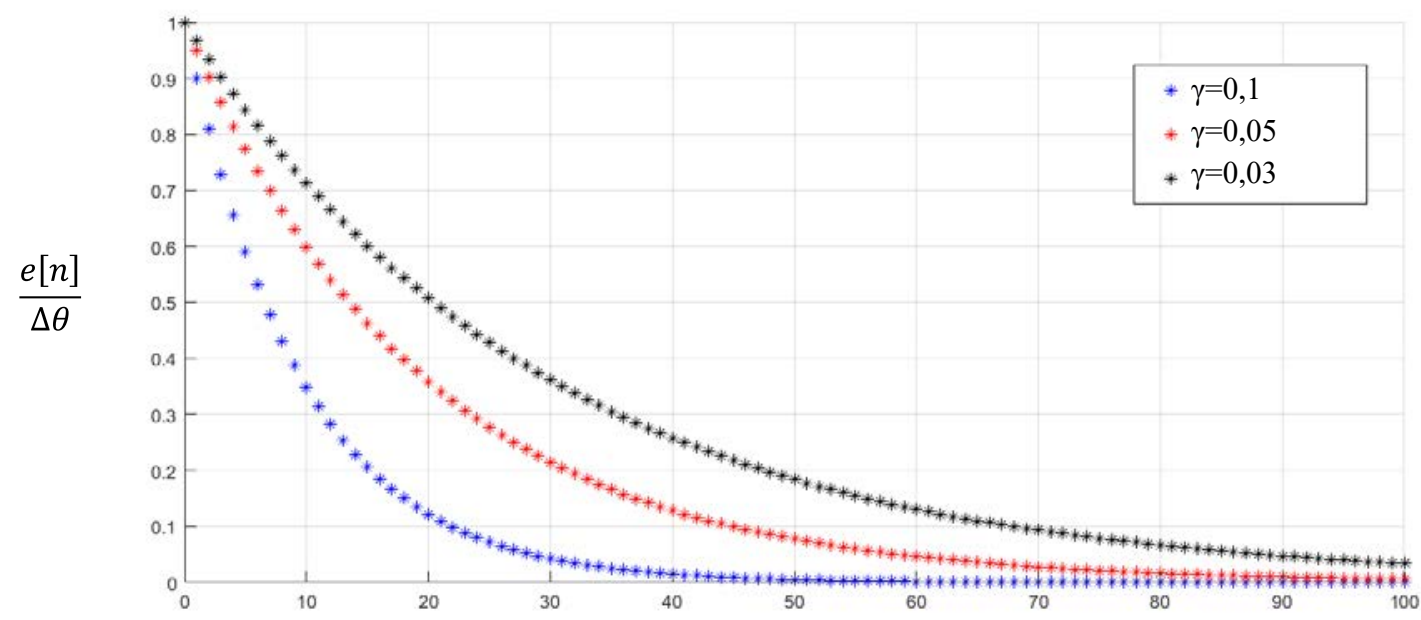

Fonte: Elaborado pelos próprios autores (2018).

Adotando-se $y=0,1$, pela fig. 7 , observa-se que, para $n>50$, o erro e[n] é praticamente desprezível, portanto o tempo de transiente da malha é aproximadamente $50 T$, em que $T$ é o período de amostragem que coincide com o período do símbolo QPSK.

\subsubsection{Análise estatística do estimador de fase}

Considerando que o sinal na entrada da Malha de Costa é dado pela eq. (16), sendo a variância do erro de fase da malha é dada por (WILLIAN, C. L.): 


$$
\sigma^{2} \hat{\theta}=\frac{1}{j 2 \pi} \oint_{|z|=1} F(z) F\left(z^{-1}\right) z^{-1} \frac{R_{z q}(z)}{A^{2}} d z
$$

em que $R_{z q}(z)$ é a transformada $z$ da função de autocorrelação da parte imaginária do ruído $z[n]=z_{i}[n]+j z_{q}[n]$ na entrada da malha e $A^{2}=2$ corresponde à potência de $s[n]$. Como $z[n]$ é um processo gaussiano e branco, com densidade espectral de potência $2 N_{0}$, tem-se:

$$
R_{z q}(z)=N_{0} .
$$

Considerando que a energia de bit do sinal equivalente em banda passante é $E_{b}=1 / 2$, usando-se a eq. (31) na eq. (30), resulta:

$$
\sigma^{2} \hat{\theta}=\frac{N_{0}}{4 E_{b}} \frac{1}{j 2 \pi} \oint_{|z|=1} F(z) F\left(z^{-1}\right) z^{-1} d z=\frac{1}{4\left(\frac{E_{b}}{N_{0}}\right)} I,
$$

em que:

$$
\mathrm{I}=\frac{1}{j 2 \pi} \oint_{|z|=1} F(z) F\left(z^{-1}\right) z^{-1} d z .
$$

Da equação (33), para $F(z)$ dado pela eq. (24) e $\gamma=0,1$, têm-se:

$$
\mathrm{I}=\frac{\gamma^{2}}{1-(\gamma-1)^{2}}=0,526
$$

Portanto, a variância do erro de fase da Malha de costa é:

$$
\sigma^{2} \hat{\theta}=\frac{0,526}{4\left(\frac{E_{b}}{N_{0}}\right)} \text {. }
$$

Para a condição de $E_{b} / N_{0}=10 \mathrm{~dB}$, a variância do erro do estimador de fase é aproximadamente igual a $1,3 \times 10^{-3} \mathrm{rad}^{2}$. o que resulta em uma perda desprezível na performance do sistema (MEYR H. et al., 1998).

\section{Resultados e discussão}

O sistema OFDM foi simulado, em várias condições de operação, com o objetivo de avaliar o seu desempenho em termos da taxa de erro de bit (BER) em função da relação sinal-ruído expressa pela relação $E_{b} / N_{0}$. Testou-se o receptor operando apenas com o estimador de frequência, ou com o estimador de fase, e também funcionando com os dois estimadores. Para realização dos testes foram utilizados três diferentes valores de CFO. Também foram levantadas as curvas da variância do erro dos estimadores de frequência e fase, bem como a curva de aprendizado da malha de Costa.

A lista a seguir apresenta resumidamente os principais parâmetros utilizados nas simulações:

- Taxa de bits: $32 \mathrm{Mbps}$

- Frequência de amostragem: $16 \mathrm{MHz}$

- Quantidade de portadoras: 16

- Espaçamento de frequência entre as subportadoras $(\Delta f): 1 \mathrm{MHz}$

- Relação sinal ruído $\left(E_{b} / N_{0}\right)$ : de 0 a $20 \mathrm{~dB}$

- Desvio de fase: $\pi / 6$ radianos

- Desvio de frequência normalizado $(\varepsilon): 0,1 ; 0,01$ e 0,001.

- Prefixo Cíclico $\left(N_{g}\right): 4$.

- Comprimento da média móvel do estimador de frequência $(K): 10^{4}$. 


\subsection{Taxa de Erro de Bit}

A fig. 8 mostra as curvas de BER do sistema para os diferentes valores de $\varepsilon$ quando não há qualquer tipo de correção no desvio de frequência. As três curvas estão sobrepostas e bem distantes da curva teórica. Podemos concluir que, sem tratamento do desvio de frequência do sinal, o desempenho do sistema é completamente degradado.

Figura 8 - BER do sistema quando o mesmo funciona sem estimadores de frequência e fase.

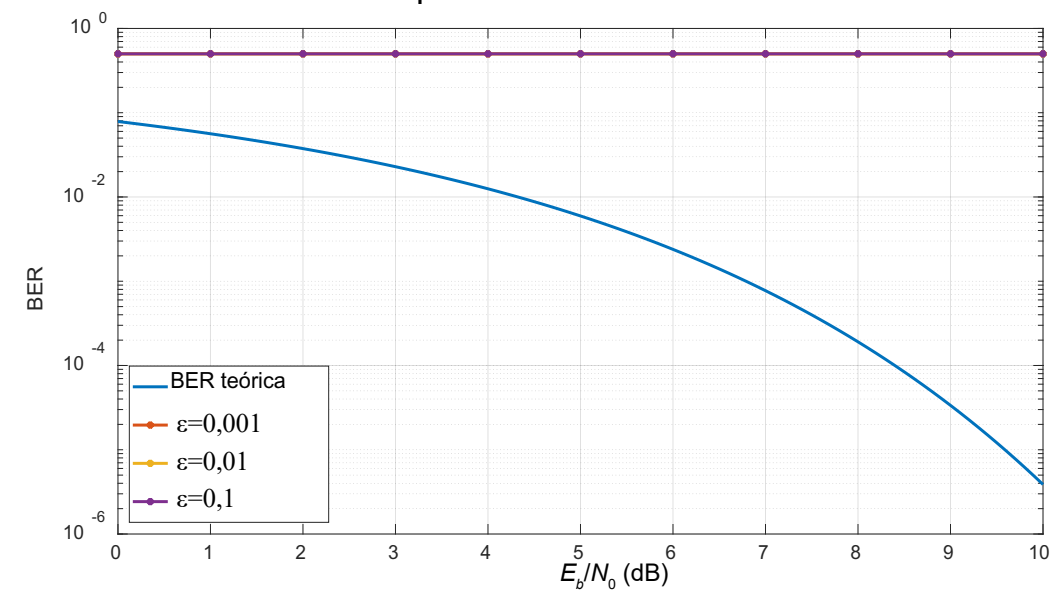

Fonte: Elaborado pelos próprios autores (2018).

O desempenho do sistema quando somente o estimador de frequência é inserido no receptor está ilustrado na fig. 9. Apesar de haver alguma melhoria na taxa de erro para $\varepsilon=0,001$, o seu desempenho ainda está bastante comprometido. Isto ocorre porque, apesar da atuação do estimador de frequência, o erro de fase residual é suficientemente grande para causar erro na decisão. Para $E_{b} / N_{0}<5 \mathrm{~dB}$, a potência de ruído é tão grande que a presença do estimador de frequência se torna praticamente indiferente em termos de melhoria na taxa de erro de bits. Por outro lado, para valores de $E_{b} / N_{0}$ próximos de $10 \mathrm{~dB}$, a pouca interferência do ruído no sinal transmitido faz com que o estimador de frequência consiga obter alguma melhoria, embora ainda insuficiente. Para $\varepsilon=0,1$, mesmo em valores de $E_{b} / N_{0}$ próximos de $10 \mathrm{~dB}$, o desvio de frequência é grande o suficiente para fazer com que o estimador de não consiga obter alguma melhoria.

Figura 9 - BER do sistema quando o mesmo funciona com o Estimador de Frequência.

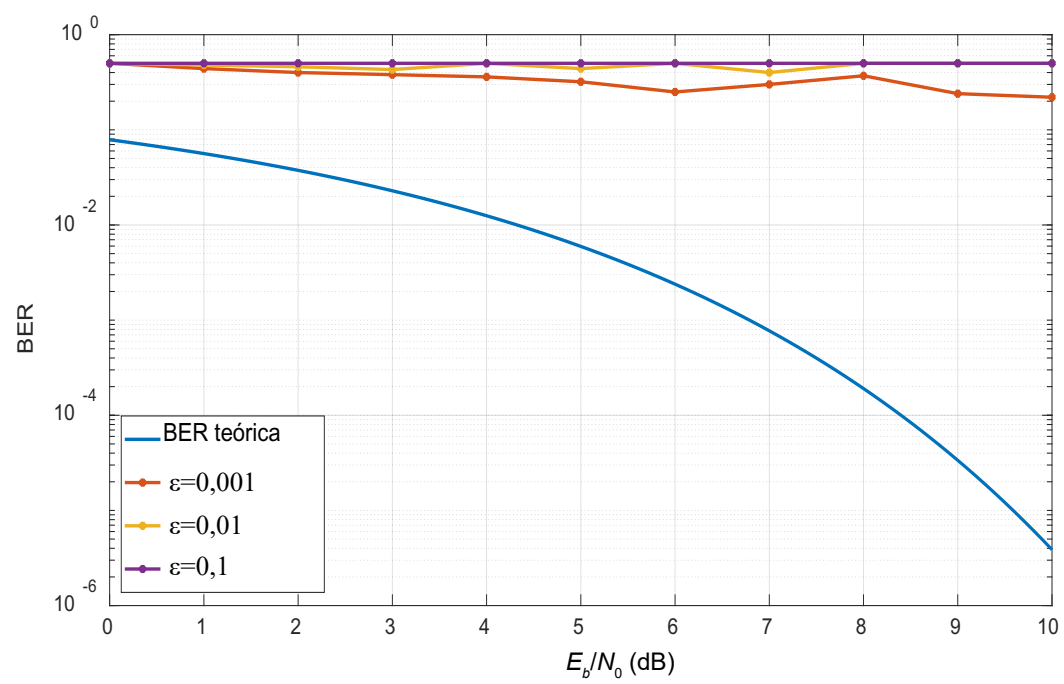

Fonte: Elaborado pelos próprios autores (2018). 
Mostra-se na fig. 10 o desempenho do sistema com o bloco Malha de Costa funcionando e o estimador de frequência desativado. Nesse caso, como o estimador de frequência não está presente, cabe ao estimador de fase cancelar tanto o offset de fase como de frequência. Observa-se nos resultados que, quando $\varepsilon=0,001$, a Malha de Costa consegue recuperar a fase e frequência de forma que a BER do sistema se aproxima bastante do valor teórico. No entanto, para $E_{b} / N_{0} \leq 1 \mathrm{~dB}$, observa-se que a taxa de erro permanece elevada. Isto ocorre porque, nessas condições de $E_{b} / N_{0}$, o nível de ruído inserido no sinal transmitido faz com que a Malha de Costa perca o lock e não consiga fazer o rastreamento da fase do sinal de entrada. Por consequência, perde o sincronismo de fase com o sinal recebido.

Pode-se observar o mesmo fenômeno quando $\varepsilon=0,01$. No entanto, como o desvio de frequência maior nessa situação, a perda do rastreamento ocorre para $E_{b} / N_{0} \leq 2 \mathrm{~dB}$. A perda de lock da malha está diretamente relacionada com o valor de $y$ adotado. Como mostrado na fig. 7 , quanto maior for $\gamma$, mais rápido o erro fase converge para zero. Contudo a malha fica mais susceptível a perda de sincronismo. Assim, têm-se uma relação de compromisso entre a velocidade de convergência da malha e a sua capacidade de rastreamento (GARDNER, F. M., 2005).

Já para $\varepsilon=0,1$, o desvio de frequência se torna maior do que o estimador de fase consegue suportar em termos de rastreamento, portanto, a informação do sinal transmitido é perdida, e, como resultado, têm-se uma elevada taxa de erro. Esse resultado demonstra a grande susceptibilidade da Malha de Costa ao valor do desvio de frequência, sendo capaz de corrigir apenas pequenos desvios de frequência. Como conclusão parcial, pode-se deduzir que, com os dois estimadores trabalhando em conjunto, o sistema deve obter ótimas taxa de erro de bits (desde que o estimador de fase esteja em sincronismo), pois o desvio de frequência imposto pelo canal será atenuado pelo estimador de frequência, e o estimador de fase, por sua vez, vai eliminar o desvio residual, recuperando a fase do sinal transmitido.

Figura 10 - BER do sistema quando o mesmo funciona com Malha

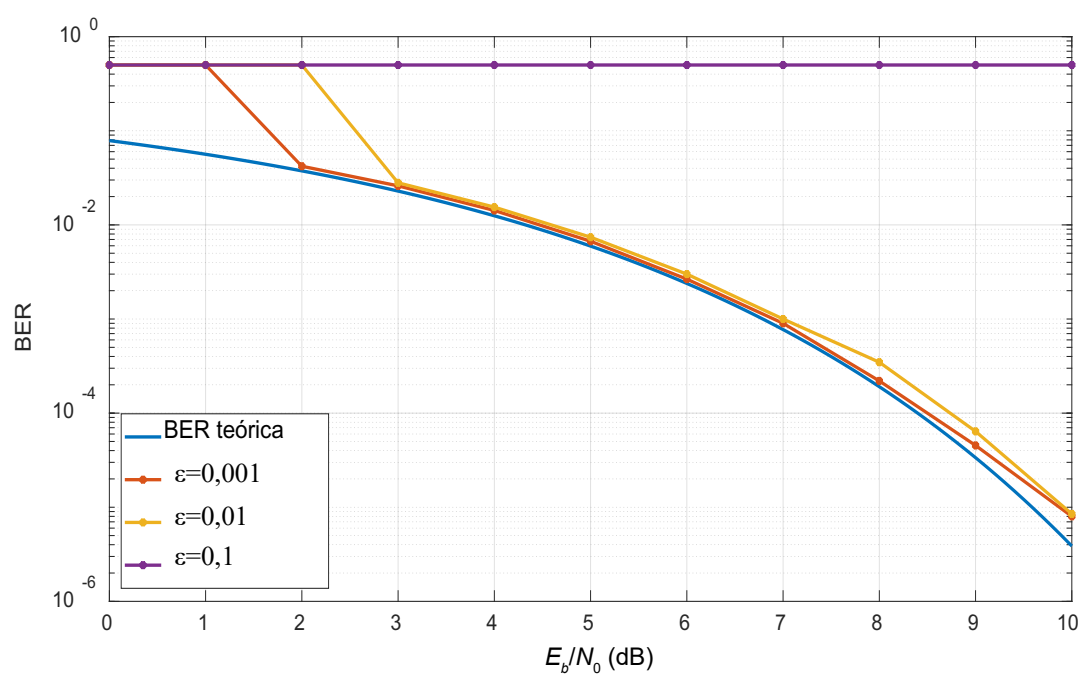

Fonte: Elaborado pelos próprios autores (2018).

As curvas de BER do sistema quando os dois circuitos de sincronismo operam simultaneamente estão indicadas na fig. 11. Como explicado anteriormente, em condições de relação sinal-ruído muito baixas, a Malha de Costa perde o sincronismo e o desempenho do sistema é degradado. Porém, quando $E_{b} / N_{0} \geq 2$ $\mathrm{dB}$, o sistema se mostrou robusto, funcionando muito bem mesmo para $\varepsilon=0,1$. Essa característica se deve ao fato do estimador de frequência realizar uma correção grosseira do desvio e o estimador de fase precisar corrigir apenas o erro de frequência residual. Como as situações de $E_{b} / N_{0}=0$ e $E_{b} / N_{0}=1 \mathrm{~dB}$ são extremas e de pouca serventia na prática, pode-se afirmar que, quando os dois estimadores operam simultaneamente, os efeitos do desvio de fase e frequência são praticamente mitigados. 
Figura 11 - BER do sistema quando o mesmo funciona com o Estimador de Frequência e Malha de Costa.

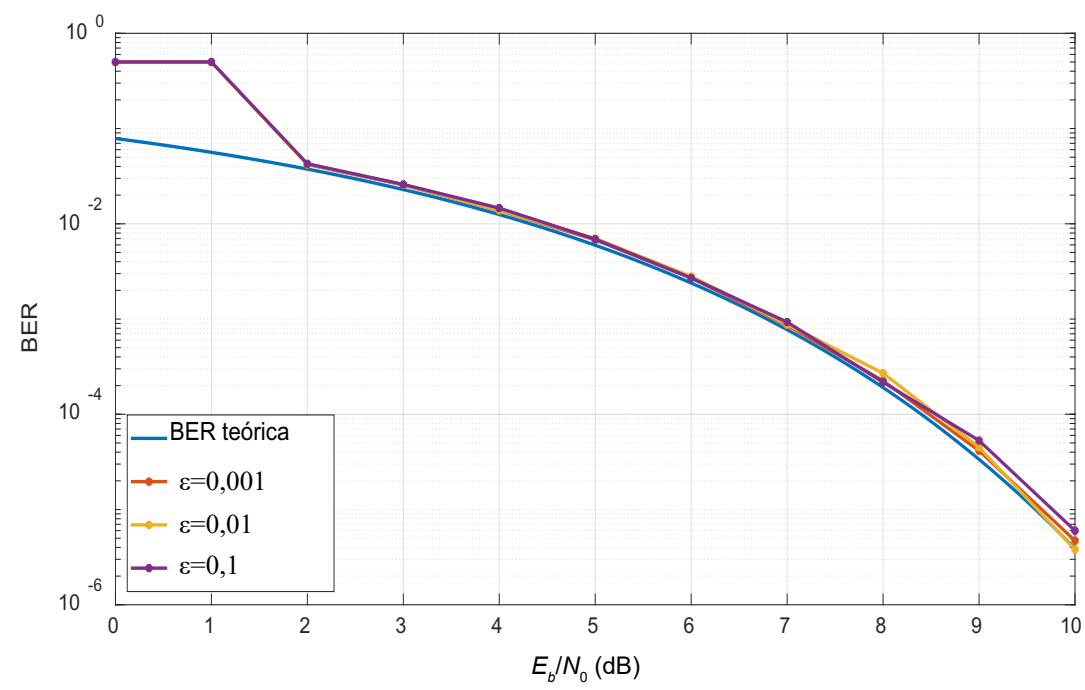

Fonte: Elaborado pelos próprios autores (2018).

\subsection{Variância da estimativa de frequência}

A figura 13 mostra o desempenho do estimador de frequência em termos da variância do valor estimado $\left(\sigma_{\hat{\varepsilon}}{ }^{2}\right)$ em função da relação $E_{b} / N_{0}$. Também foi apresentada como referência a curva do limite de Cramer-Rao modificado para essa variância, que é dado por (MENGALI; D'ANDREA, 2013):

$$
\operatorname{MCRB}(\varepsilon)=\frac{3}{4 \pi^{2} K^{3} T^{2}} \frac{1}{E_{b} / N_{0}} \frac{1}{\Delta f^{2}},
$$

em que $k$ é o comprimento da média temporal do estimador de frequência, e $T$ é o período do sinal transmitido, que é igual a $0,05 \mu s$.

Figura 12 - Variância do estimador de Frequência.

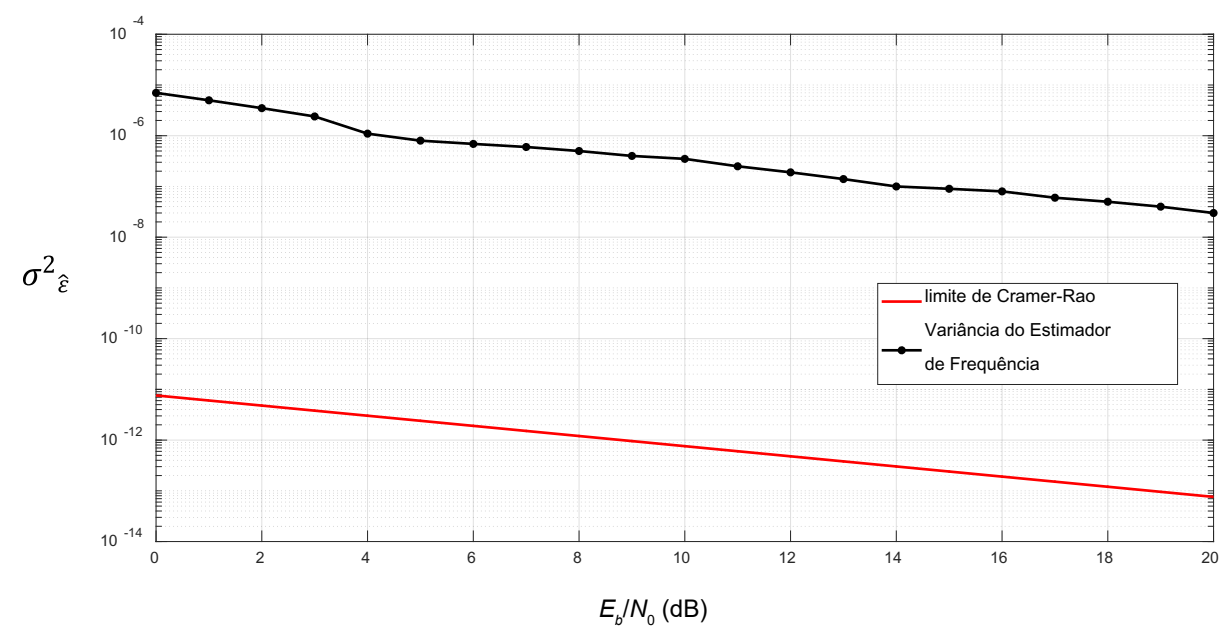

Fonte: Elaborado pelos próprios autores (2018).

Observa-se uma discrepância considerável entre a variância do estimador de frequência e o limite de Cramer-Rao, mostrado na fig. 13. Isto já era esperado, pois a arquitetura de estimador adotado não é ótima. Entretanto, mesmo com essa imprecisão, a Malha de costa funcionando em conjunto é capaz de cancelar o offset residual, de forma que praticamente não há impacto no desempenho do sistema. 


\subsection{Tempo de aquisição da Malha de costa}

A figura 12 mostra o gráfico da curva de aprendizagem da Malha de costa para $E_{b} / N_{0}=20 \mathrm{~dB}$. Observase que o tempo de convergência é cerca $30 \mu s$, que corresponde a $50 T$, concordando com o valor de projeto determinado na secção 3 .

Figura 13 - Curva de aprendizagem para $E_{b} / N_{0}=20 \mathrm{~dB}$.

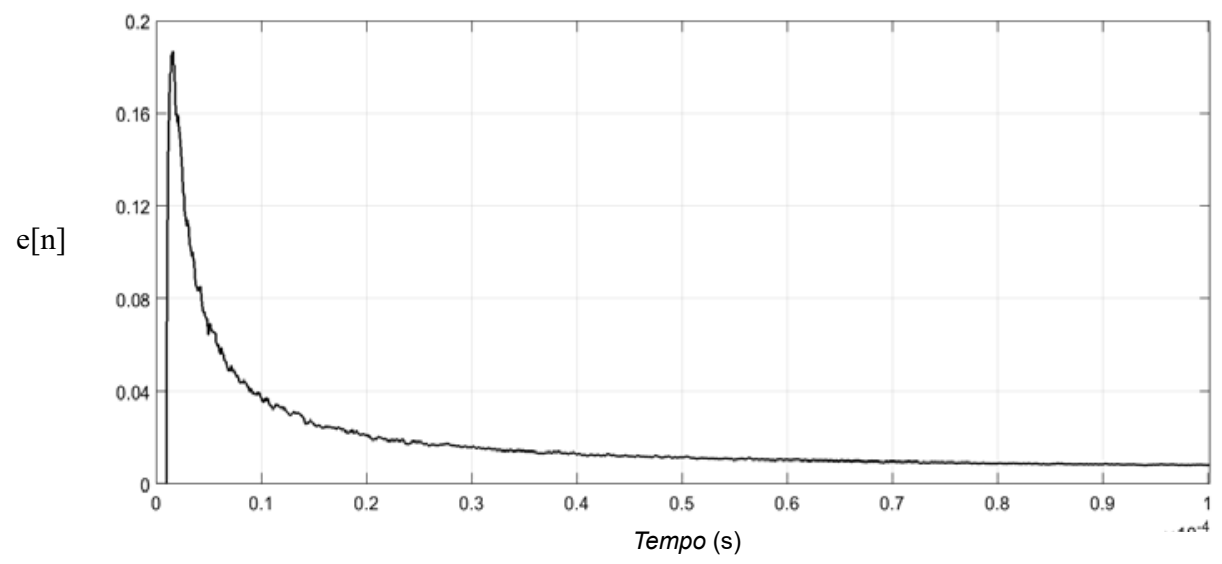

Fonte: Elaborado pelos próprios autores (2018).

\subsection{Variância da estimativa de fase}

A curva da variância da fase estimada pela Malha de Costa $\sigma_{\hat{\theta}}^{2}$ está mostrada na fig. 14. Também foram traçadas a curva teórica da variância, conforme a eq. (35), e o limite de Cramer-Rao modificado para a variância de fase, expresso por (MENGALI; D'ANDREA, 2013):

$$
\operatorname{MCRB}(\theta)=\frac{1}{4 L_{0}} \frac{1}{E_{b} / N_{0}} \text { e } \frac{1}{L_{0}}=\frac{1}{j 2 \pi} \oint_{|z|=1} F(z) F\left(z^{-1}\right) z^{-1} d z .
$$

É interessante notar que as três curvas coincidem. Da eq. (37) e eq. (35) está evidente que o valor da variância do erro de fase para a Malha de Costa é igual ao limite de Cramer-Rao. Por outro lado, a concordância da curva simulada indica a grande precisão do modelo computacional. Como já previsto na secção 2, para $E_{b} / N_{0}=10 \mathrm{~dB}$, a variância do erro do estimador de fase é de fato aproximadamente igual a $1,3 \times 10^{-3} \mathrm{rad}^{2}$. Essa imprecisão provoca uma perda desprezível no desempenho do sistema.

Figura 14 - Variância do estimador de fase.

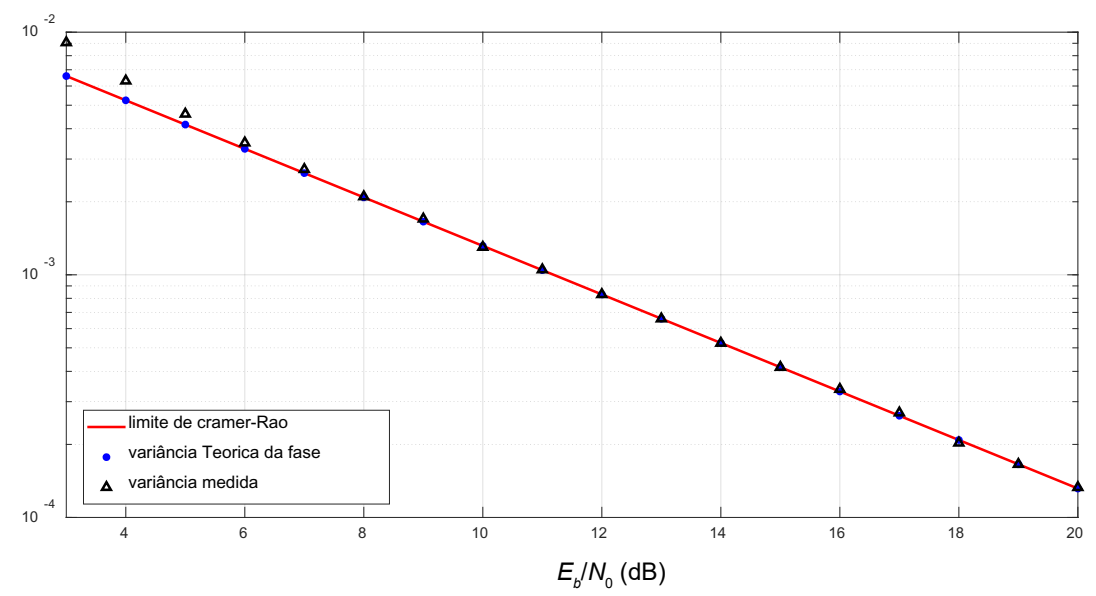

Fonte: Elaborado pelos próprios autores (2018). 


\section{Conclusão}

Neste trabalho apresentou-se o projeto de um sistema de comunicação OFDM em que o receptor é dotado de estimador de frequência e de estimador de fase para sincronização das subportadoras do sistema. Todos os componentes do sistema foram detalhados por meio de diagramas de blocos e equações. O estimador de fase é implementado por meio de uma Malha de Costa discreta, cujos parâmetros são determinados analiticamente. Avaliou-se, através de simulação computacional, o desempenho do sistema em termos de taxa de erro de bit quando existe offset de frequência. Também avaliou-se os estimadores de frequência e de fase. A curva de aprendizado da Malha de Costa, obtida via simulação, é também apresentada.

Com os dois estimadores funcionando conjuntamente, e com $E_{b} / N_{0} \geq 2 \mathrm{~dB}$, a BER do sistema praticamente atinge o desempenho teórico. Portanto, nessas condições, a imprecisão da estimativa de frequência e fase tem impacto nulo no desempenho do sistema.

Quando o receptor está equipado apenas com o estimador de frequência, o desempenho do sistema fica completamente degradado, mesmo para $\varepsilon=0,001$. Por outro lado, se o receptor utiliza somente o estimador de fase, para as condições de $\varepsilon=0,001$ e $E_{b} / N_{0} \geq 2 \mathrm{~dB}$, ou $\varepsilon=0,01$ e $E_{b} / N_{0} \geq 3 \mathrm{~dB}$, tem-se a taxa de erro de bit do sistema próximo do limite teórico.

À Malha de Costa, apesar de sua simplicidade, funciona muito bem com variância do estimador de fase atingindo o limite de Cramer-Rao. O tempo de convergência da malha, mostrada na fig. 12, comportou-se conforme previsto.

Como perspectiva futura para esta pesquisa, pode-se avaliar o desempenho dos estimadores para canais dispersivos.

\section{Agradecimentos}

Este trabalho teve o suporte financeiro parcial do Conselho Nacional de Desenvolvimento Científico e Tecnológico (CNPq).

\section{Referências}

GARDNER, F. M. Phaselock techniques. New York: John Wiley \& Sons, 2005.

LI, Jian; LIU, Guoqing; GIANNAKIS, Georgios B. Carrier frequency offset estimation for OFDM-based WLANs. IEEE Signal Processing Letters, Mississippi, v. 8, n. 3, p. 80-82, 2001.

LINDSEY, William C.; CHIE, Chak Ming. A survey of digital phase-locked loops. Proceedings of the IEEE, New York, v. 69, n. 4, p. 410-431, 1981.

LUCENA, A. M. P. et al. Fully digital BPSK demodulator for satellite supressed carrier telecommand system. International Journal of Satellite Communications and Networking, Nova Jersey, v. 35, n. 4, p. 359-374, 2017. DOI: https://doi.org/10.1002/sat.1188

MA, Xiaoqiang; KOBAYASHI, Hisashi; SCHWARTZ, Stuart C. Effect of frequency offset on BER of OFDM and single carrier systems. In: PERSONAL, INDOOR AND MOBILE RADIO COMMUNICATIONS, 14., 2003, Pequim. Proceedings... IEEE, 2003, p. 2239-2243. DOI: 10.1109 / PIMRC.2003.1259065

MENGALI, Umberto; D’ANDREA, Aldo N. Synchronization techniques for digital receivers. New York: Springer Verlag, 2013.

MEYR, H.; MOENECLAEY, M.; FECHTEL, S. Digital communication receivers: synchronization, channel estimation, and signal processing. New York: John Wiley \& Sons, 1998.

MOHSENI, Saeed; MATIN, Mohammad A. Study of the estimation techniques for the Carrier Frequency 
Offset (CFO) in OFDM systems. International Journal of Computer Science and Network Security, Seoul, v. 12, n. 6, p. 73, 2012.

MORELLI, Michele; KUO, C.-C. Jay; PUN, Man-On. Synchronization techniques for orthogonal frequency division multiple access (OFDMA): a tutorial review. Proceedings of the IEEE, New York, v. 95, n. 7, p. 1394-1427, 2007.

PRIYA, C. G; VASUMATHI, A. M. Frequency Synchronization in OFDM System. Journal of Signal and Information Processing, Amsterdam, v. 4, n. 03, p. 138-143, 2013. DOI: 10.4236 / jsip.2013.43B024_

SATHANANTHAN, Kanagaratnam; TELLAMBURA, Chintha. Performance analysis of an OFDM system with carrier frequency offset and phase noise. In: VEHICULAR TECHNOLOGY CONFERENCE VTC 2001 Fall. IEEE VTS, 54., Atlantic, 2001. Proceedings... IEEE, 2001. p. 2329-2332.

SHAYAN, Y. R.; LE-NGOC, T. All digital phase-locked loop: concepts, design and applications. In: IEE Proceedings F-Radar and Signal Processing. IET, 1989. p. 53-56.

WEINSTEIN, Stephen B. The history of orthogonal frequency-division multiplexing [History of Communications]. IEEE Communications Magazine, v. 47, n. 11, 2009. p. 26-35. DOI: 10.1109 / MCOM.2009.5307460

WU, Fan; ABU-RGHEFF, Mosa Ali. Time and frequency synchronization techniques for OFDM systems operating in gaussian and fading channels: a tutorial. In: ANNUAL POSTGRADUATE SYMPOSIUM ON THE CONVERGENCE OF TELECOMMUNICATIONS, NETWORKING AND BROADCASTING, 8., Liverpool, Reino Unido, 2007. Proceedings...Liverpool, p. 263-268, 2007. Disponível em: <http://www.cms.livjm.ac.uk/ pgnet2007/Proceedings/Papers/2007-042.pdf>. Acesso em: 14 maio 2017.

Sobre os autores

\section{Rubem Vasconcelos Pacelli}

Graduação em Engenharia Eletrônica pela Universidade de Fortaleza - Unifor. Durante o período de graduação, estagiou na Armtec, onde trabalhou no projeto e layout de PCB (Printed Circuit Board). Foi monitor voluntário da disciplina de teoria de controle, onde desenvolveu métodos de ensino em Matlab/Simulink.Foi bolsista CNPq na área de telecomunicações, onde estuda técnicas de modulações, processamento de sinais e comunicação espacial. Atualmente é aluno de mestrado do Programa de Pós-Graduação em Teleinformática (PPGETI) na UFC, onde realiza estudos na área de comunicação espacial e dispositivos FPGA's. Tem interesse em sistemas de telecomunicações, processamento digital de sinais e sistemas embarcados: microcontroladores; FPGA e DSP; projeto e layout de PCI.

\section{Antônio Macilio Pereira de Lucena}

Graduado em Engenharia Eletrônica pelo Instituto Tecnológico de Aeronáutica (ITA), São José dos Campos-SP, Brasil, 1980, mestre em eletrônica e telecomunicações espaciais pelo Instituto Nacional de Pesquisas Espaciais (Inpe), São José dos Campos-SP, Brasil, 1986, e doutor em engenharia de teleinformática pela Universidade Federal do Ceará (UFC), Fortaleza-CE, Brasil, 2006. Trabalha no Inpe desde 1983, onde desenvolveu vários projetos nas áreas de telecomunicações espaciais, eletrônica e radioastronomia. Desde 2007 é também professor da Universidade de Fortaleza (Unifor). Seus atuais interesses em pesquisa incluem técnicas de sincronização, telecomunicações espaciais, processamento de sinal, processamento de imagem e teoria de comunicação.

Recebido em: 08/02/2018

Avaliado em: 17/04/2018

Aceito em: 18/04/2018 